\title{
Corrigendum
}

\section{Corrigendum to "Clinical Characteristics of Visual Dysfunction in Carbon Monoxide Poisoning Patients"}

\author{
Wei-Kang Bi $\mathbb{D},{ }^{1,2}$ Jing-Lin Wang $\left(\mathbb{D},{ }^{3}\right.$ Xu-Dong Zhou $\mathbb{D D}^{2,4}$ Ze-Kun Li ${ }^{(D)},{ }^{2,5}$

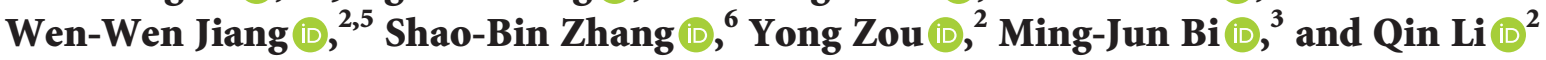 \\ ${ }^{1}$ Weifang Medical University, Weifang, Shandong 261042, China \\ ${ }^{2}$ Department of Integration of Chinese and Western Medicine, Yantai Yuhuangding Hospital Affiliated to Qingdao University, \\ Yantai, Shandong 264000, China \\ ${ }^{3}$ Emergency Center, Yuhuangding Hospital Affiliated to Qingdao University, Yantai, Shandong 264000, China \\ ${ }^{4}$ Shandong Wendeng Osteopathic Hospital, Yantai, Shandong 264000, China \\ ${ }^{5}$ Centre of Integrated Chinese and Western Medicine, School of Basic Medicine, Qingdao University, Qingdao, \\ Shandong 266071, China \\ ${ }^{6}$ Weifang Eye Hospital, Weifang, Shandong 261000, China
}

Correspondence should be addressed to Shao-Bin Zhang; zhangsb518@163.com and Qin Li; liqin701015@163.com

Received 2 December 2020; Accepted 2 December 2020; Published 18 January 2021

Copyright (c) 2021 Wei-Kang Bi et al. This is an open access article distributed under the Creative Commons Attribution License, which permits unrestricted use, distribution, and reproduction in any medium, provided the original work is properly cited.

In the article titled "Clinical Characteristics of Visual Dysfunction in Carbon Monoxide Poisoning Patients" [1], there was a spelling error in author Jing-Lin Wang's name in the author list, where "Jin-Lin Wang" should have read as "Jing-Lin Wang." This is corrected in the author list above.

\section{References}

[1] W.-K. Bi, J.-L. Wang, Xu-D. Zhou et al., "Clinical characteristics of visual dysfunction in carbon monoxide poisoning patients," Journal of Ophthalmology, vol. 2020, Article ID 9537360, 8 pages, 2020. 